\title{
Calidad de la Orientación en la Facultad de Educación (Universidad de Santiago de Compostela)
}

\author{
The quality of Guidance in the Faculty of Educational Sciences \\ (University of Santiago de Compostela)
}

\author{
$M^{a}$ Cristina Ceinos Sanz, Miguel Anxo Nogueira Pérez \\ Departamento de Métodos de Investigación y Diagnóstico en Educación. Facultad de Ciencias de la Educación. \\ Universidad deSantiago de Compostela
}

\begin{abstract}
Resumen
De los cambios producidos en la sociedad actual, cobran especial relevancia los acontecidos en el sistema universitario, contexto en el que de cara a favorecer la capacitación integral del alumnado, debe considerarse el proceso de maduración personal que todo estudiante experimenta, así como la inclusión de la Orientación en dicha etapa. Por tanto, el principal objetivo del presente trabajo se centra en analizar las acciones de Orientación desarrolladas con alumnado de último curso de la Facultad de Ciencias de la Educación (Universidad de Santiago de Compostela) con la pretensión de formular planteamientos de mejora en las acciones desarrolladas.

Palabras clave: Sociedad de la Información y de la Comunicación, Orientación Universitaria, Orientación estudiantil, Evaluación y Mejora de la Orientación.
\end{abstract}

\begin{abstract}
From the changes which are produced in the current society, the ones happened in the university system are particularly relevant, context in which to favour the integral capacity of students, the personal maturity process that every student experiments must be considered as well as the Guidance inclusion in such stage. Therefore, the main objective of the present study is focused on analyzing the Guidance actions developed with final-year students of the Faculty of Educational Science (University of Santiago de Compostela) with the aim of formulating proposals for improvement in the developed actions.

Key Words: Information and Communication Society, University Guidance, Student Guidance, Guidance Evaluation and Improvement.
\end{abstract}

La sociedad actual, conocida, entre otras denominaciones, como Sociedad de la Información y del Conocimiento, se caracteriza, entre otros aspectos, por su complejidad, por el gran número de transformaciones acontecidas a todos los niveles, así como por la necesidad de adquirir y asumir competencias y perfiles profesionales más actualizados y acordes con las demandas planteadas hoy en día.

Entre los principales factores que originaron el entramado social actual, el cual se consolida a comienzos del presente siglo, cobra especial relevancia la introducción y desarrollo de herramientas y recursos tecnológicos, así como las mejoras derivadas de estos, aspectos todos ellos causantes de la auténtica revolución tecnológica experimentada en los últimos tiempos.
No obstante, de forma complementaria, destaca, también, la influencia ejercida por múltiples factores en la totalidad de ámbitos y sectores, los cuales dieron lugar a importantes transformaciones en el mundo laboral, en el ámbito social, cultural y educativo, así como en las estructuras económicas.

Es en dicho contexto, en el que el sistema educativo en general y la Universidad en particular se encuentran inmersos, por lo que su principal cometido, a diferencia de aquel tradicional, propio de momentos anteriores, centrado en la creación y transmisión de cultura y capacitación de profesionales, ha de ampliarse, ofreciendo, en consecuencia, una respuesta a la totalidad de demandas y exigencias planteadas por la sociedad actual, al tiempo que ofrecer a los sujetos la formación y capacitación necesaria para poder desempeñar cometidos profesionales y sociales en el entorno en el que conviven y al cual pertenecen (Álvarez, Asensio, Forner \& Sobrado, 2006).

En dicho contexto, cobra especial relevancia el papel a desempeñar por la Orientación en la etapa universitaria, a pesar de que su concreción en la práctica difiera del principal cometido de dicha disciplina. Al respecto, en lo que al papel de la Orientación Universitaria se refiere, cabe apuntar el gran número de investigaciones existentes, las cuales, prácticamente, en su totalidad, coinciden en señalar la existencia de ciertas tendencias en lo que a Orientación se refiere en dicha etapa educativa, las cuales se concretan del siguiente modo: (Rodríguez, 1997; Sánchez, 1998; López \& Oliveros, 1999; Álvarez, V., García-Jiménez, E., Gil-Flores, J. \& Romero, S., 2000; Vidal, Díez \& Vieira, 2002; Valverde, 2004; García \& Sanz, 2006; García, 2010 y Lobato \&Ilvento, 2013): falta de modelos y experiencias de Orientación consolidadas; heterogeneidad de actividades ofertadas y estrategias empleadas; predominio y supremacía de un enfoque reactivo y de un modelo de intervención a través de servicios; intervenciones esporádicas y puntuales de carácter, fundamentalmente, informativo y centradas en el ámbito académico-profesional; insuficiencia de recursos materiales; escasez de personal con formación específica en la atención al alumnado en los Servicios de Orientación, así como iniciativas llevadas a cabo en base a motivos coyunturales y no en función de estudios sistemáticos de necesidades. 
Así pues, tomando como referente los principales resultados derivados de las investigaciones anteriormente citadas, se precisa realizar un replanteamiento de la Orientación en el contexto universitario, considerando la pertinencia de crear servicios y fomentar programas de Orientación dentro de las Universidades, superar la función informativa y avanzar hacia un concepto más amplio y diverso, en el que se engloben $\mathrm{y}$ tengan cabida los aspectos económicos, personales y profesionalizadores, así como la totalidad de funciones inherentes a esta disciplina (Suárez, 2013). Así pues, con dicha pretensión, el presente trabajo centra su atención en analizar las acciones de Orientación llevadas a cabo con el alumnado de último curso de las diferentes Titulaciones impartidas en la Facultad de Ciencias de la Educación (Campus Vida) de la Universidad de Santiago de Compostela con el objetivo básico de conocer y analizar una realidad concreta, así como realizar y formular, en caso de necesidad, propuestas de mejora en materia de Orientación.

\section{Método}

La metodología de investigación empleada, tomando como referente el problema de investigación propuesto, los objetivos formulados, así como la naturaleza del fenómeno a investigar, fue de carácter descriptivo, realizando un estudio tipo encuesta, por considerarse eficaz para realizar un estudio de carácter exploratorio y/o describir un fenómeno o realidad concreta, en este caso centrado, fundamentalmente, en conocer, describir y valorar las acciones de Orientación llevadas a cabo con el alumnado de último curso de la Facultad de Ciencias de la Educación (Campus Vida) de la Universidad de Santiago de Compostela, así como plantear ciertos elementos de reflexión, debate y discusión que sirvan como referente en la planificación de posteriores trabajos de investigación y en la propuesta de planteamientos de mejora.

\section{Participantes}

La muestra definitiva estuvo conformada por 160 estudiantes de último curso de la Licenciatura en Psicopedagogía, Grado en Educación Social y Grado en Pedagogía durante el curso académico 2013-2014.

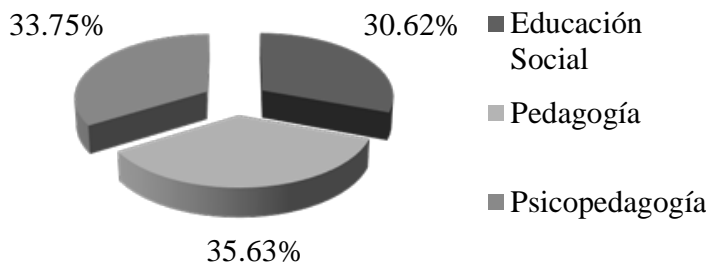

Figura 1. Distribución de la muestra según Titulación cursada en la Facultad de Ciencias de la Educación de la Universidad de Santiago de Compostela (Campus Vida). Fuente: Elaboración propia

\section{Instrumento}

Como instrumento de recogida de información, se empleó un cuestionario, a través del cual se recabó información referida al perfil de la muestra, así como a las acciones de Orientación llevadas a cabo con este colectivo durante sus estudios universitarios. Para ello, se diseñó un cuestionario compuesto por un total de 17 ítems, estructurados en dos bloques claramente diferenciados: perfil de la muestra (7 ítems) y acciones de Orientación desarrolladas con el alumnado de la Facultad de Ciencias de la Educación (Campus Vida) (10 ítems).

\section{Procedimiento}

El proceso de recogida de información tuvo lugar durante el desarrollo de sesiones expositivas de diferentes materias de carácter obligatorio en las diferentes Titulaciones impartidas en la Facultad de Ciencias de la Educación (Campus Vida) de la Universidad de Santiago de Compostela durante el curso académico 2013-2014, procediendo al análisis de la información recabada, fundamentalmente de carácter cuantitativo, a través del paquete estadístico SPSS (versión 19.0).

\section{Resultados}

En el presente trabajo, se recoge la síntesis de los principales resultados obtenidos en un estudio de carácter cuantitativo realizado en la Facultad de Ciencias de la Educación de la Universidad de Santiago de Compostela (Campus Vida), cuyo principal cometido se centra en describir y analizar los principales datos identificativos de la muestra, así como conocer las acciones de Orientación recibidas por los estudiantes de dicha institución durante la realización de sus estudios universitarios.

Así pues, en lo que al perfil de la muestra encuestada se refiere, cabe apuntar que nos encontramos ante un colectivo de estudiantes de las diferentes Titulaciones impartidas en la Facultad de Ciencias de la Educación (Campus Vida) de la Universidad de Santiago de Compostela, mayoritariamente femenino, menor de veinticinco años, procedente del Bachillerato u otra Titulación Universitaria (no debe olvidarse que la Licenciatura en Psicopedagogía constituía una Titulación de Segundo Ciclo), desempleado, no inscrito en el Servicio Público de Empleo y con intención de seguir formándose o buscar empleo al término de sus estudios (ver tabla 1). 
Tabla 1.

Caracterización de la muestra encuestada

\begin{tabular}{|c|c|}
\hline VARIABLES & PORCENTAJES \\
\hline Sexo & $\begin{array}{l}\text { Hombres: } 10 \% \\
\text { Mujeres: } 90 \%\end{array}$ \\
\hline Edad & $\begin{array}{l}\text { Menores de } 25 \text { años: } 82.5 \% \\
\text { Entre } 25-29 \text { años: } 12.5 \% \\
\text { De } 30 \text { en adelante: } 5 \%\end{array}$ \\
\hline Titulación a Cursar & $\begin{array}{l}\text { Grado en Pedagogía: 35.63\% } \\
\text { Grado en Educación Social: } 30.62 \% \\
\text { Licenciatura en Psicopedagogía: } 33.75 \%\end{array}$ \\
\hline Estudios Previos realizados & $\begin{array}{l}\text { Bachillerato: } 50.63 \% \\
\text { Ciclos Formativos de Grado Superior: } 8.12 \% \\
\text { Otra Titulación Universitaria: } 41.25 \%\end{array}$ \\
\hline Situación laboral actual & $\begin{array}{l}\text { En activo: } 16.25 \% \\
\text { Desempleado: } 83.75 \%\end{array}$ \\
\hline Inscripción en el Servicio Público de Empleo & $\begin{array}{l}\text { No: } 80.62 \% \\
\text { Sí, como demandante de empleo: } 15 \% \\
\text { Sí, como solicitante de mejora de empleo: } 4.38 \%\end{array}$ \\
\hline Intención al finalizar los estudios & $\begin{array}{l}\text { Seguir formándose: } 45 \% \\
\text { Buscar empleo: } 38.75 \% \\
\text { Seguir formándose y buscar empleo: } 13 \% \\
\text { Mantener el empleo actual y seguir formándose: } 1.25 \% \\
\text { Otras intenciones (sin especificar): } 2 \%\end{array}$ \\
\hline
\end{tabular}

Fuente: Elaboración propia

Por otra parte, en lo que respecta a las acciones de orientación recibidas por el alumnado durante su estancia en la Facultad de Ciencias de la Educación (Campus Vida), cabe apuntar, en una primera aproximación, que nos encontramos ante un colectivo que, en su mayoría, ha recibido acciones de Orientación durante su trayectoria académica, las cuales, de forma mayoritaria, fueron desarrolladas con escasa frecuencia por parte del profesorado de la Facultad, estudiantes de cursos superiores o profesionales externos a la Facultad, normalmente, durante el último curso de carrera y bajo demanda del propio alumnado.

Por otra parte, en lo que respecta al Servicio de Orientación propio de la Universidad, puede afirmarse que éste es desconocido para la mitad de los sujetos encuestados, siendo utilizado en pocas o algunas ocasiones por parte del alumnado que tuvo conocimiento del mismo durante sus estudios. En lo que respecta al tipo de consulta realizada y al tipo de acciones de Orientación recibidas, cabe apuntar que éstas se centraron, mayoritariamente, en cuestiones de carácter informativo, relacionadas, fundamentalmente, con el ámbito académico-profesional. No obstante, a pesar de la insatisfacción manifestada por el colectivo de estudiantes en lo que respecta a las acciones de Orientación recibidas durante sus estudios universitarios, estos resaltan la gran utilidad de las iniciativas puestas en marcha por el Servicio de Orientación propio de la Universidad de Santiago de Compostela.
A la vista de los resultados expuestos en la tabla 2, cabe apuntar la necesidad de llevar a cabo con el alumnado de las diferentes Titulaciones impartidas en la Facultad de Ciencias de la Educación de la Universidad de Santiago de Compostela (Campus Vida) un mayor número de actuaciones en materia de Orientación por parte de la Facultad y del Servicio de Orientación Universitaria, las cuales han de implementarse con mayor frecuencia, así como realizar una mayor divulgación y difusión de las mismas y del Servicio en aras de favorecer el conocimiento de éstas y su integración en la cultura y comunidad universitaria.

Complementariamente, en lo que respecta a las acciones llevadas a cabo, debe reconocerse la supremacía de acciones puntuales y esporádicas, siempre bajo demanda del estudiante, con predominio de un enfoque reactivo y restrictivo, ya que, en la mayor parte de las ocasiones, se implementan acciones centradas en el ámbito académico-profesional, con carácter, fundamentalmente, informativo, descuidando, en consecuencia, la dimensión personal de la Orientación, así como otras múltiples funciones de dicha disciplina (diagnóstica, formativa, de ayuda y consejo, de consulta, de organización y planificación, así como de evaluación e investigación) (Ceinos, 2008).

Finalmente, según los resultados obtenidos, a pesar del escaso conocimiento y pequeña participación de la muestra en el Servicio de Orientación propio de la Universidad, la utilidad concedida al mismo es positiva, 
por lo que destaca la importancia que el alumnado concede a este tipo de iniciativas y la necesidad, en consecuencia, de realizar mayores esfuerzos por potenciar dicho Servicio, así como difundir y promover su actividad.

Tabla 2.

Principales características de las acciones de Orientación desarrolladas con el alumnado de Ciencias de la Educación

\begin{tabular}{|c|c|}
\hline VARIABLES & PORCENTAJES \\
\hline $\begin{array}{l}\text { Destinatarios de acciones de orientación durante los } \\
\text { estudios universitarios }\end{array}$ & $\begin{array}{l}\text { Sí: } 75 \% \\
\text { No: } 25 \%\end{array}$ \\
\hline Frecuencia de las acciones de Orientación & $\begin{array}{l}\text { A menudo: } 0.83 \% \% \\
\text { En alguna ocasión: } 31.67 \% \\
\text { En pocas ocasiones: } 67.5 \%\end{array}$ \\
\hline Temporalización de las acciones de Orientación & $\begin{array}{l}\text { Al comienzo de los estudios universitarios: } 0 \% \\
\text { Durante la realización de estudios universitarios: } 14.5 \% \\
\text { Al finalizar los estudios universitarios: } 85.5 \%\end{array}$ \\
\hline Agentes responsables & $\begin{array}{l}\text { Profesorado: } 46.25 \% \\
\text { Profesionales externos a la Facultad: } 24.38 \% \\
\text { Estudiantes de cursos superiores: } 16.25 \% \\
\text { Servicio de Orientación de la Universidad: } 5 \% \\
\text { Otros: } 4.37 \% \\
\text { No responden: } 3.75 \%\end{array}$ \\
\hline Origen de la acción orientadora & $\begin{array}{l}\text { Bajo demanda del alumnado: } 72.4 \% \\
\text { Por iniciativa del profesorado: } 26.4 \% \\
\text { Por iniciativa del Servicio de Orientación Universitario: } 1.2 \%\end{array}$ \\
\hline $\begin{array}{l}\text { Conocimiento del Servicio de Orientación durante los } \\
\text { estudios }\end{array}$ & $\begin{array}{l}\text { Sí: } 47.5 \% \\
\text { No: } 52.5 \%\end{array}$ \\
\hline Frecuencia de uso del Servicio & $\begin{array}{l}\text { A menudo: } 0 \% \\
\text { Bastante a menudo: } 6.58 \% \\
\text { Algo a menudo: } 15.79 \% \\
\text { Poco a menudo: } 21.05 \% \\
\text { Nunca: } 56.58 \%\end{array}$ \\
\hline $\begin{array}{l}\text { Tipo de consulta realizada al Servicio de Orientación } \\
\text { Universitaria }\end{array}$ & $\begin{array}{l}\text { Información Académica y Profesional: } 62.58 \% \\
\text { Orientación Profesional: } 16.36 \% \\
\text { Orientación Académica: } 12.22 \% \\
\text { Orientación Personal: } 2.75 \% \\
\text { Otras: } 6.09 \%\end{array}$ \\
\hline $\begin{array}{l}\text { Utilidad de las acciones del Servicio de Orientación } \\
\text { Universitaria }\end{array}$ & $\begin{array}{l}\text { Mucha: } 27.27 \% \\
\text { Bastante: } 42.42 \% \\
\text { Alguna: } 24.24 \% \\
\text { Poca: } 6.07 \% \\
\text { Ninguna: } 0 \%\end{array}$ \\
\hline $\begin{array}{l}\text { Satisfacción con la Orientación recibida durante los } \\
\text { estudios }\end{array}$ & $\begin{array}{l}\text { Mucha: } 7.20 \% \\
\text { Bastante: } 10.15 \% \\
\text { Alguna: } 18.40 \% \\
\text { Poca: } 39.50 \% \\
\text { Ninguna: } 24.75 \%\end{array}$ \\
\hline
\end{tabular}

Fuente: Elaboración propia

\section{Discusión}

En consecuencia, a tenor de los resultados obtenidos, así como del análisis e interpretación realizado al respecto, cabe señalar que la muestra encuestada se encuentra inmersa en una realidad que poco difiere de lo ya reflejado por múltiples investigaciones realizadas en lo que a Orientación Universitaria se refiere, mencionadas algunas de ellas en páginas precedentes.

Así pues, se está en condiciones de afirmar que nos encontramos ante un colectivo de estudiantes universitarios que ha recibido pocas acciones de Orientación durante su trayectoria universitaria, fundamentalmente, con carácter informativo, implementadas, mayoritariamente, de manera puntual y 
esporádica en el último curso de sus estudios y desarrolladas, fundamentalmente, por el profesorado de la Titulación, estudiantes de cursos superiores y/o agentes externos a la Facultad, aspectos todos ellos que reflejan la escasa visibilidad del Servicio de Orientación Universitario, apenas conocido por un colectivo importante de la muestra encuestada.

Es por esto que urge considerar la etapa universitaria como un contexto ideal en el que deben y han de llevarse a cabo un mayor número de acciones orientadoras, siendo preciso, en consecuencia, establecer pautas de actuación, las cuales se centren, mayoritariamente, en el establecimiento de un modelo y estrategias de Orientación consolidadas, contextualizadas en un marco común y con predominancia de un enfoque preventivo, en detrimento de aquél otro de carácter reactivo; diseñar e implementar acciones de Orientación con carácter continuo y en base a estudios sistemáticos de necesidades; integrar la dimensión personal, académica y profesional en las acciones de Orientación de cara a satisfacer todas y cada una de las necesidades que el alumnado manifieste; contar con los recursos materiales necesarios y con personal especializado; favorecer la creación de servicios de Orientación propios en la Facultad de Ciencias de la Educación, complementarios a los de la Universidad, así como potenciar un Plan de Acción Tutorial, aspectos todos ellos que permitirán la orientación y seguimiento del alumnado desde su ingreso hasta la finalización de sus estudios, así como impulsar y visibilizar las experiencias y acciones llevadas a cabo por la totalidad de agentes implicados en la Facultad de Ciencias de la Educación con el propósito de favorecer, de este modo, el desarrollo pleno de los estudiantes, así como la inclusión de dichas iniciativas en la comunidad y cultura universitaria.

Finalmente, a la vista de las recomendaciones anteriores, debe prestarse la atención requerida por la Orientación en el contexto universitario, así como suplir y dar respuesta a las carencias y necesidades orientadoras existentes en el contexto universitario en general y en la Facultad de Ciencias de la Educación de la Universidad de Santiago de Compostela (Campus Vida) en particular, de cara a favorecer, en parte, el cumplimiento de la atención personalizada, orientación y asesoramiento que, de acuerdo con las directrices actuales, constituye un factor de innovación y calidad en las instituciones universitarias.

\section{Referencias}

Álvarez, P., Asensio, I., Forner, A.,\&Sobrado, L. M. (2006). Los planes de acción tutorial en la Universidad. En T. Escudero \& A. D. Correa (Coords.), Investigación en innovación educativa: algunos ámbitos relevantes (pp. 147-206). Madrid: La Muralla.

Álvarez, V., García-Jiménez, E., Gil-Flores, J.,\& Romero, S. (2000). La orientación en la universidad en el contexto de una docencia de calidad. En J. Ruiz \& A. Medina, Orientación educativa e intervención psicopedagógicas, I Jornadas Andaluzas de Orientación y Psicopedagogía (pp. 209-252). Jaén: Servicio de Publicaciones de la Universidad de Jaén.
Ceinos, C. (2008). Diagnóstico de las competencias de los orientadores laborales en el uso de las Tecnologías de la Información y de la Comunicación. Tesis Doctoral. Universidad de Santiago de Compostela.

García, B (2010). La tutoría en la Universidad de Santiago de Compostela: Percepción y valoración del alumnado y profesorado. Tesis Doctoral. Universidad de Santiago de Compostela.

García, E.,\& Sanz, R. (2006). Nuevas estrategias de apoyo a los estudiantes en la Universidad. En T. Escudero \& A. D. Correa (Coords.), Investigación en innovación educativa: algunos ámbitos relevantes (pp. 207-268). Madrid: La Muralla.

Lobato, C.,\&Ilvento, M. C. (2013). La orientación y tutoría universitaria: una aproximación actual. Revista de Docencia Universitaria, 11(2), 17-25.

López, E.,\& Oliveros, L. (1999). La tutoría y la orientación en la Universidad. Revista Española de Orientación y Psicopedagogía, 10(17), 83-98.

Rodríguez, S. (1997). Orientación Universitaria y evaluación de la calidad. En P. Apodaca \& C. Lobato (Eds.), Calidad en la universidad: Orientación y Evaluación (pp. 23-52). Barcelona: Laertes.

Sánchez, M. F. (1998). Las funciones y necesidades de orientación en la Universidad: un estudio comparativo sobre las opiniones de universitarios y profesionales. Revista de Orientación y Psicopedagogía, 9(15), 87-107.

Suárez, B. (2013). Servicios de Orientación profesional universitarios: estudio descriptivo. Revista de Docencia Universitaria, 11(2), 27-42.

Valverde, A. (2004). Orientación universitaria: evaluación de un sistema de estudiantes mentores. Tesis doctoral. Universidad de Sevilla.

Vidal, J., Díez, G.,\& Vieira, M ${ }^{\mathrm{a}}$ J. (2002). La oferta de los servicios de orientación en las universidades españolas. Revista de Investigación Educativa, 20(2), 431-448. 